\title{
TRAPPED OSCILLATIONS IN THE CHROMOSPHERE IN THE PRESENCE OF A MAGNETIC FIELD
}

\author{
Y. NAKA GAWA \\ High Altitude Observatory, National Center for Atmospheric Research, ${ }^{*}$ Boulder, Colo., U.S.A.
}

\begin{abstract}
In the presence of a magnetic field, three types of magnetoatmospheric waves - magnetoacoustic mode, magnetogravitational mode, and hydromagnetic mode - can propagate in a stratified atmosphere, in contrast to the propagation of two types of atmospheric waves - acoustic mode and gravitational mode - in the absence of a magnetic field. The exact manner of propagation of the magnetoatmospheric wave is extremely complex, and most studies have been confined to certain specific circumstances, such as an isothermal atmosphere permeated by a uniform magnetic field (McLellan and Winterberg, 1968; Bel and Mein, 1971; Michalitsanos, 1973), and atmosphere in magnetohydrostatic equilibrium (Yu, 1965; Chen and Lykoudis, 1972)
\end{abstract} and the propagation across a density discontinuity (Stein, 1971).

In a previous paper (Nakagawa et al., 1973) a general method of determining the trapped magnetoatmospheric waves was described, and in another paper (Nakagawa, 1973) possible interpretation in terms of such theoretical results of the observed modulations of chromospheric oscillations over the magnetically active regions of the Sun was discussed. Therefore we present a brief summary of the physically significant results of previous studies, with discussions focused on the validity of assumptions and the results obtained.

The main effect of a magnetic field is the modulation of the period of trapped oscillations, and this effect can be examined by means of the diagnostic diagrams. It was shown (Nakagawa et al., 1973) that in a horizontal magnetic field except for the magnetoacoustic waves, most of the waves are trapped, and that in a general magnetic field most of the waves of frequencies below the local Brunt-Vaisala frequency are trapped (contrary to the non-magnetic case in which those waves are propagating gravitational waves). Further it was shown (Nakagawa, 1973) that a number of satisfactory agreements can be obtained between the theoretical and observational results. Notably, it was suggested that the short period oscillations of around $170 \mathrm{~s}$ reported by Elliot (1969) and Bhatnagar and Tanaka (1972) inside of supergranulation could be identified with the trapped magnetoatmospheric waves traveling along the magnetic lines, as such short period oscillations cannot result from the magnetogravitational waves traveling perpendicular to the magnetic field. Similarly, it was pointed out that the observed period of 600-700 s by Orrall (1966) and Blondel (1971) in bright facular regions could be identified with the trapped oscillations in the presence of a vertical magnetic field, together with the observed period of $900 \mathrm{~s}$ by Orall (1966), Elliot (1969) and Cha (1970) in the supergranulation boundaries.

\footnotetext{
* The National Center for Atmospheric Research is sponsored by the National Science Foundation.
} 
In summary, the need of radiative-hydrodynamic both observational and theoretical studies for the understanding of the chromospheric oscillations is stressed, since most of the theoretical studies have been confined only to the examinations of adiabatic small amplitude perturbations.

\section{References}

Bel, N. and Mein, P.: 1971, Astron. Astrophys. 11, 234.

Bhatnagar, A. and Tanaka, K.: 1972, Solar Phys. 24, 87.

Blondel, M.: 1971, Astron. Astrophys. 10, 342.

Cha, J. Y. M.: 1970, Thesis, University of Hawaii.

Chen, C.-J. and Lykoudis, P. S.: 1972, Solar Phys. 25, 380.

Elliot, I.: 1969, Solar Phys. 6, 28.

McLellan, A. and Winterberg, F.: 1968, Solar Phys. 4, 401.

Michalitsanos, A. G.: 1973, Solar Phys. 30, 47.

Nakagawa, Y.: 1973, Solar Phys. 33, 87.

Nakagawa, Y., Priest, E. R., and Wellck, R. E.: 1973, Astrophys. J. 184, 931.

Orrall, F. Q.: 1966, Astrophys. J. 143, 917.

Stein, R. F.: 1971, Astrophys. J. Suppl. 22, 419 (No. 192).

Yu, C. P.: 1965, Phys. Fluids 8, 650.

\section{DISCUSSION}

Stix: Would you clarify one point. Is in each of your layers the Alfven velocity and the density constant. If so, is this consistent with the stratification?

Nakagawa: The density falls off exponentially within each layer with a constant scale height. Thus the Alfvén velocity also changes. However, in the analysis the Alfvén velocity is assumed constant in each layer as we have found that superposition of such solutions gives an adequate approximation to the exact solution.

Souffrin: I am concerned about the identification of the slow mode with the magnetogravitational mode. In a homogeneous medium with a vertical magnetic field you already have Alfvén waves, a slow mode and a fast mode. In the presence of gravitation there is a new possibility for waves.

Nakagawa: No the dispersion relations still allow only three wave modes.

Souffrin: If you just suppress the gravitation you have three possible wave modes. Do you mean that when you add the gravitation you must modify these three modes?

Nakagawa: The dispersion relations still allow only three wave modes. The addition of a gravitational field modifies the wave mode but does not introduce a possibility for a new type of wave to exist. How you identify each of these modes with the modes in the non-gravitational case is of course somewhat open to question. 